\title{
Analysis of Deep Level Parameters in Irradiated Silicon Detectors
}

\author{
C. Betancourt, G. Alers, N. Dawson, M. Gerling, R. F. Hurley, H. F.-W. Sadrozinski, S. Sattari \\ Santa Cruz Institute for Particle Physics, University of California, Santa Cruz 1156 High St., Santa Cruz, Ca 95064, \\ phone: (831)-459-3567,email: cbetanco@ucsc.edu
}

\begin{abstract}
Both the collected charge and capacitance of a silicon detector is proportional to the depleted depth of the space charge region. The presence of deep levels introduces a frequency dependence in the measured capacitance, and this frequency dependence can be corrected for by analyzing the data using admittance spectroscopy. An equivalent circuit for a PN junction containing deep levels is used to accurately model deep level response. Activation energy, majority carrier cross section and the concentration of active deep levels at the edge of the space charge region is determined. Space charge region depth as a function of the applied voltage is extracted for various detectors irradiated with neutrons and pions. When possible, depleted depths are compared to charge collection measurements.
\end{abstract}

PACS: 29.40.Wk; 29.40.Gx; 85.30.De, 83.30.Kk

Keywords: silicon detector, deep level, admittance spectroscopy, radiation damage, charge collection, PN junction

\section{INTRODUCTION}

$\mathrm{T}^{\mathrm{s}}$ he main operational parameter of a silicon strip detector is the bias voltage dependence of the charge, collected at the electrodes when a particle is traversing the device. We have shown before that the bias dependence of the collected charge, called $C C E(V)$, and of the reciprocal capacitance from $C-V$ measurements are identical, since both are dependent on the thickness of the space charge region layer [1]. This is true as long as the detector depleted from the front side near the implant. During irradiation, the bulk of some detectors go through type inversion, switching the junction to the back side. This causes charge traveling from the back to the front of the detector to get trapped before it can be received, leading to a reduction in collected charge. Unless depletion happens from the front, CV and CCE measurements should not agree. Evidence of a double junction $[2,3]$ in some devices could cause a detector to deplete from the front even in a type inverted detector, causing CV and $\mathrm{CCE}$ to agree. Care should also be taken to correct for the frequency dependence of $C-V$ measurements by taking into account deep level response to the AC signal.

For a minimum ionizing particle (mip), the number of electron/hole pairs is generated uniformly in the detector with $73 \mathrm{e} / \mu \mathrm{m}$ [4], and thus the most probable charge generated is linearly proportional to the thickness of the depleted region. For this reason,

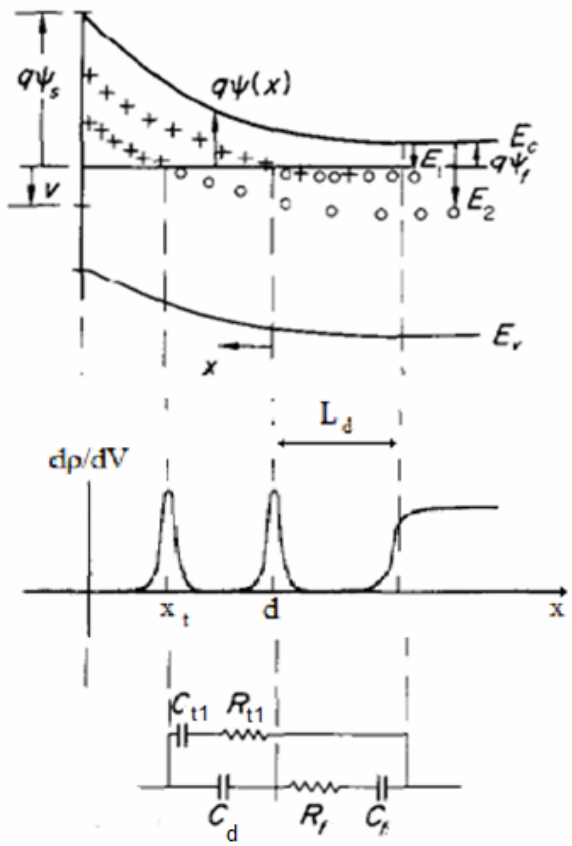

Fig. 1. Energy band diagram for a junction containing deep level with the corresponding equivalent circuit [8]. The generation/recombination contribution to the admittance of each deep level can be modeled as a capacitor and resistor in series, which is then in parallel to the capacitance of the device. the profile of the collected charge for mips, evaluated as a function of the applied reverse voltage $(C C E(V))$, should exhibit the same trend as the reciprocal of the capacitance $(1 / C(V))$, since the capacitance of a parallel-plate capacitor varies inversely with its thickness. (This is important strictly true only as long as trapping effects can be neglected, which become very important at fluences above $10^{15} \mathrm{neq} / \mathrm{cm}^{2}$ [5].) Contrary to un-irradiated detectors, where the doping density is a constant as a function of the depth, irradiated detectors exhibit a non-uniform space charge distribution depending on the bias voltage $[2,3]$.

It is known that irradiation alters the electrical properties of silicon by the introduction of deep levels within the band gap [6]. There have been several studies on the effects of deep levels on the capacitance and admittance of semiconductor junction devices $[7,8,9]$. As a result, the true space charge region capacitance is convoluted by the frequency response of deep levels.

This paper is presented as follows. Section II will describe experimental set up and data, Section III describes Admittance measurements and relation to the space charge depth. Section IV presents results. Section VI gives a summary of results and conclusions. 


\section{EXPERIMENTAL SET UP AND DATA}

$\mathrm{C}-\mathrm{V}$ measurements have been performed on silicon pad detectors produced of both p-on-n and n-on-p and FZ and MCZ material. The un-irradiated sensors were produced by MICRON at Sussex, UK, on $300 \mu \mathrm{m} \mathrm{FZ} \mathrm{wafers} \mathrm{of} \mathrm{about} 20 \mathrm{k} \Omega \mathrm{cm}$ resistivity and $\mathrm{MCz}$ wafers of about $2 \mathrm{k} \Omega \mathrm{cm}$ resistivity and by Hamamatsu Photonics (HPK) at Hamamatsu City, Japan, on $330 \mu \mathrm{m}$ FZ wafers of about resistivity. The neutron irradiation was performed at Ljubljana and pion irradiation was performed at PSI in Switzerland.

The charge collection and $C-V$ measurements on microstrip detectors have been performed at SCIPP, UC Santa Cruz. The $C C E(V)$ investigations have been carried out with a ${ }^{90} \mathrm{Sr}$ beta source, described in [10]. The $C-V$ experimental set-up is based on a HP 4284A LCR meter coupled with the HP 16065A text fixture (modified to permit biasing with voltages up to $700 \mathrm{~V}$ ). Voltage sourcing and current monitoring are performed through a computer controlled Keithley $2410 \mathrm{HV}$ supply. In order to reduce the leakage current noise, $C C E(V)$ and $C-V$ measurements taken at $-20^{\circ} \mathrm{C}$. More details on the experimental system for $C C E(V)$ are given in $[1]$.

\section{Admittance Measurements \& Space Charge Depth}

There are four competing processes that are involved in generation/recombination through a deep level; emission of an electron into the conduction band, capture of an electron from the conduction band, emission of a hole into the valence band and capture of a hole from the valence band. These four processes determine the steady state filling and charge state of deep levels. When an AC signal is applied to device, deep levels respond to the signal by filling and emptying through generation /recombination. The response of the deep levels depends on the frequency used. Each deep level will respond to the AC signal independent of the other deep levels. The frequency in which the deep levels start to contribute to the capacitance is the frequency that corresponds to the emission rate of that level. Since the minority free carrier density is much smaller than the majority free carrier density, we can neglect minority carrier response to the AC signal in the bulk. The relaxation time associated with this level for n-type material is given by $[7,8]$,

$$
\tau_{t}=\left(2 e_{n, t}\right)^{-1}
$$

and for p-type

$$
\tau_{t}=\left(2 e_{p, t}\right)^{-1}
$$

where $e_{(n, p), t}$ is the emission rate for electrons and holes respectively

The frequency response of the deep levels can more easily be studies by looking at the complex admittance of a device,

$$
Y=Z^{-1}=G_{p}+j \omega C_{p},
$$

where the capacitor and resistor in series of the impedance is turned into a capacitor and conductor in parallel. In order to model the contribution to the conductance and capacitance, an equivalent circuit model taking into account the contribution of generation-recombination and crossover capacitance to the admittance must be used. An equivalent circuit for a junction containing deep levels is derived in both $[7,8]$, and displayed in Figure 1. In this model, $G_{p} / \omega$ is given by,

$$
G_{p} / \omega=\sum_{\text {traps }} C_{b t} \frac{\ln \left(1+\omega^{2} \tau_{t}^{2}\right)}{2 \omega \tau_{t}}
$$

and $C_{p}$ is given by,

$$
C_{p}=C_{h f}+\sum_{\text {traps }} C_{b t} \frac{\tan ^{-1}\left(\omega \tau_{t}\right)}{\omega \tau_{t}}
$$

Here $C_{b t}$ is the capacitance of the number of active bulk deep levels within a few kT of the crossover with the bulk Fermi level and is given by [7],

TABLE I

Deep Level Parameters

$$
C_{b t}(V)=\frac{\sqrt{2} C_{d}(V) N_{t}}{N_{t-1}}\left(1-E_{t} / E_{f}\right)^{-1 / 2}
$$

where $C_{d}(V)$ is the space charge region capacitance, $N_{t}$ is the number of active concentration of the $t^{\text {th }}$ deep level, and $\mathrm{N}_{0}$

is the original doping of the device. For low to moderate fluences $\left(<10^{15}\right.$ neq) the term $\left(1-E_{t} / E_{f}\right)^{-1 / 2}$ is taken to be close to unity. The term $C_{h f}$ is the high frequency capacitance and is given by $[7,8]$,

$$
C_{h f}=\left(1 / C_{d}+1 / C_{f}\right)
$$




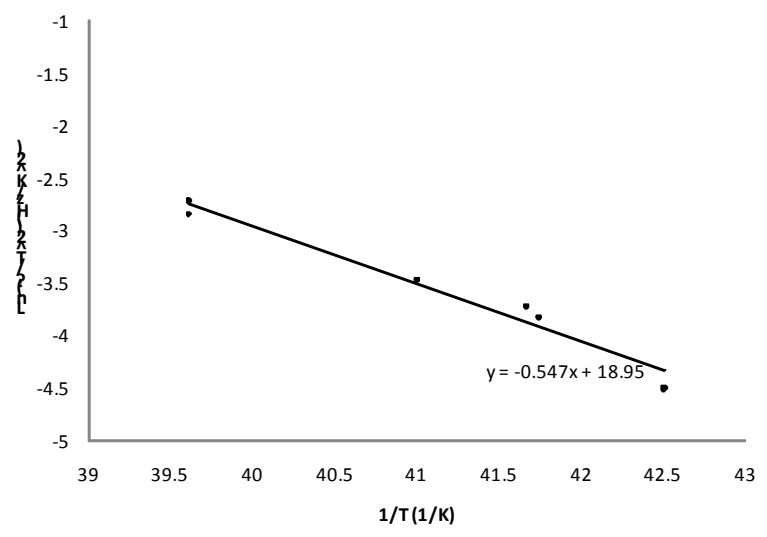

Fig. 2. Arrhenius plot for the dominant deep level in an p-on-n type FZ detector irradiated to $4.2 * 10^{\wedge} 14$ pion eq.

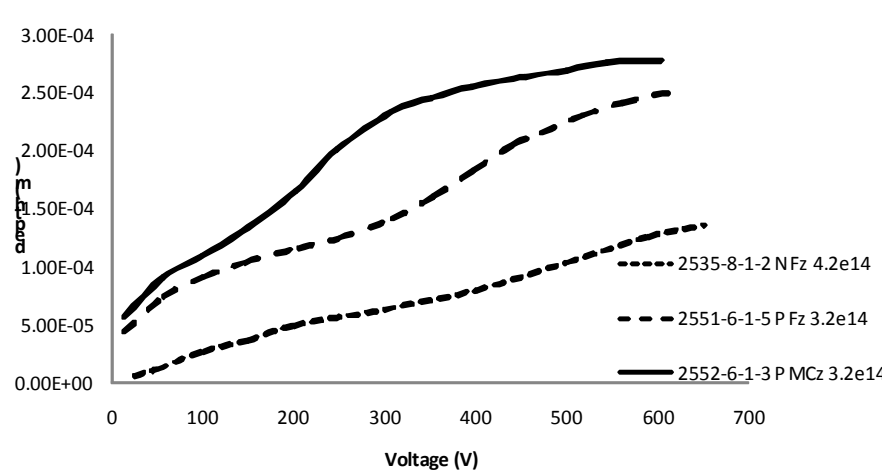

Fig. 4. Depleted depth of the space chare region for various pion detectors calculated using equation (9)

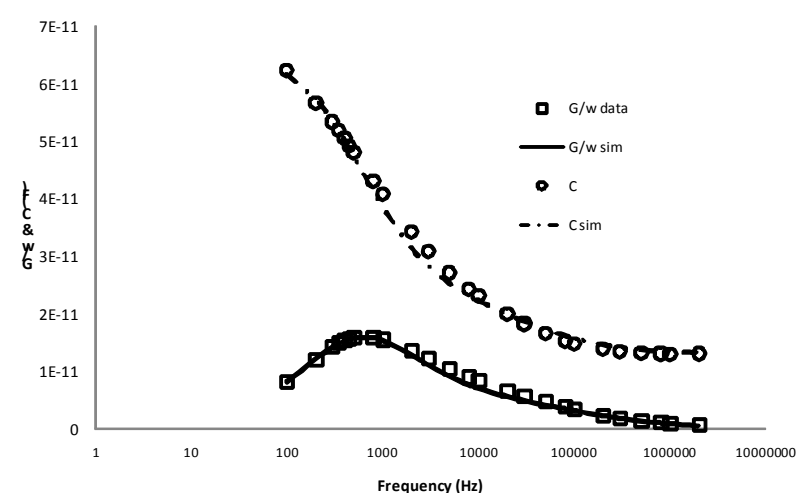

Fig. 3. Measured and simulated $\mathrm{C}$ and $\mathrm{G} / \omega$ as a function of frequency for a pion irradiated n-type $\mathrm{FZ}$ detector taken at $100 \mathrm{~V}$ and $22^{\circ} \mathrm{C}$

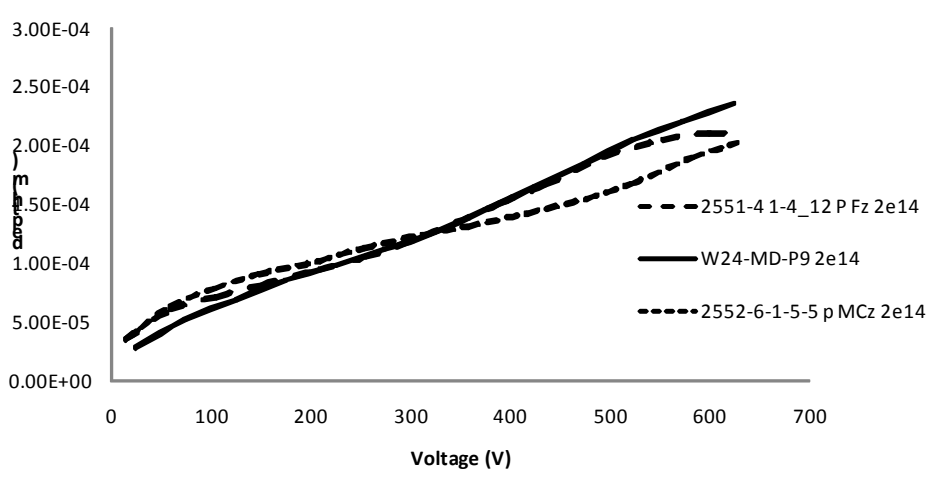

Fig. 5. Depleted depths of the space charge region for various neutron detectors calculated using equation (9)

where $C_{f}$ is the flatband capacitance associated with the free carrier concentration at the edge of the space charge region. For increasing fluences, $C_{f}$ dominates the high frequency capacitance and as a result $C_{h f}$ becomes less and less voltage dependent with increasing deep level concentration [9].

We take the capacitance of the space charge region at depletion to be

$$
C_{d}\left(V_{d e p}\right)=\varepsilon A /\left(W-L_{D}\right)
$$

where $W$ is the total width of the device and $L_{D}$ is the Debey length associated with $C_{f}$. Combining equations (7) and (8) results in

$$
d(V)=\frac{C_{b t}\left(V_{d e p}\right)}{C_{b t}(V)}\left(W-L_{d}\right)
$$

which gives the depth of the space charge region in an irradiated silicon detector.

\section{EXTRACTION OF DeEP LEVEl PARAMETERS}

Now that the effect of deep level response to the admittance is known, the energy, majority capture cross section and concentration of active deep levels within a few kT of the Fermi level can be determined. From equation (4) a maximum in $G / \omega$ curve is observed whenever

$$
\omega_{p} \tau_{t}=1.98
$$

where $\omega_{p}$ is the value of the angular frequency where the maximum occurs. From this and equations (1) and (2) the temperature dependence of the peak frequency is determined by,

$$
\omega_{p}=2(1.98) c_{n, p} N_{c, v} e^{\mp\left(E_{c, v}-E_{t}\right) / k T} \propto T^{2} e^{\mp\left(E_{c, v}-E_{t}\right) / k T}
$$

where $c_{n, p}$ are the capture probabilities for electrons and holes, $N_{c, v}$ is the density of states in the conduction and valence bands and $E_{c, v}$ are the energy of the band edges. An Arrhenius plot can then be used to determine $E_{t}$ as seen in Figure 2. Once the activation energy of the deep level is known, the majority capture cross section can easily be calculated from (11). The 


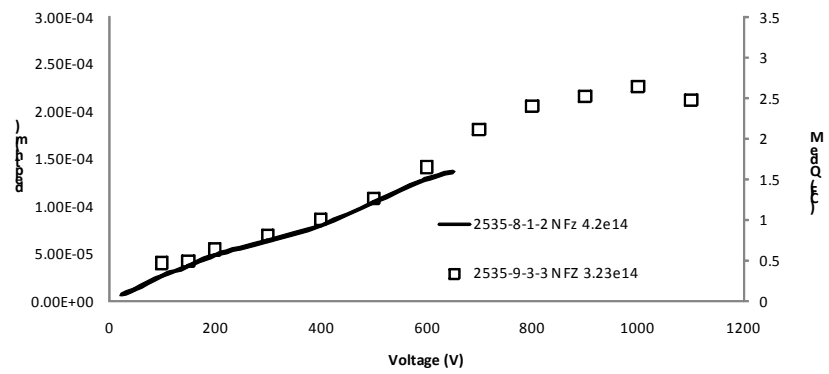

Fig. 6. Extracted depth of the space charge region compared to charge collection for p-on-n FZ pion irradiated sensors

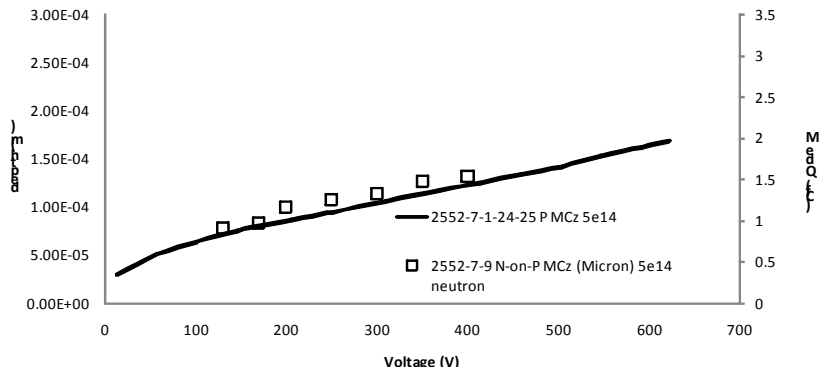

Fig. 7. Extracted depth of the space charge region compared to charge collection for n-on-p MCz neutron irradiated sensors

concentration of active deep levels within a few $\mathrm{kT}$ of the crossover point can be determined once $C_{d}$ is known, and then by plugging into equation (6).

\section{RESULTS}

Admittance measurements were taken on several pad detectors of both p-on-n and n-on-p types as well as FZ and MCz. Figure 3 displays a measured and simulated admittance curves for a p-on-n N FZ pion irradiated detector taken at a reverse bias of $100 \mathrm{~V}$ and temperature of $22^{\circ} \mathrm{C}$. The simulation was produced assuming two dominant deep levels, although one deep level was clearly more dominant than the other. Admittance measurements were only taken up to $630 \mathrm{~V}$ and for detectors that did not deplete before this, the depletion voltage was taken from previous data on corresponding detectors. Depleted depths of the space charge region for various pion irradiated sensors are displayed in Figure 4 and various neutron irradiated detectors are displayed in Figure 5.

The depth of the space charge region for several detectors of different types, irradiation, and fluences was calculated using equation (9). Charge Collection measurements are compared to the depleted depth for one p-on-n FZ pion, Figure 6, and one non-p MCz neutron, Figure 7, irradiated detector and good agreement is observed

Deep level parameters for the most dominant deep level were extracted and presented in Table 1. Energy level, majority carrier capture cross section, active concentration at the crossover, and average concentration from the depletion voltage are presented. All energies are taken with respect to the conduction band. The energy of deep level is close to midgap as expected. The concentration of active deep levels at the depletion edge $\left(N_{t}\right)$ is larger for pion than for neutron detectors, although the average density (taken from $V_{d e p}$ ) seems to be higher for neutrons. Capture cross sections are larger for neutrons than for pions.

\section{CONCLUSIONS AND SUMMARY}

We have shown that by studying the admittance of an irradiated device, important information is revealed about the most dominant deep levels introduced by irradiation. The frequency response of the deep level can be determined by constructing an equivalent circuit that takes into account generation and recombination through deep levels as well as any effect that free carriers have on the device.

Once the admittance is modeled using equations (4) and (5), deep level parameters can be extracted from fitting the model to the data. The amplitude of the peak in the $G / \omega$ is directly proportional to both the depletion capacitance and the number of active deep levels within a few $\mathrm{kT}$ of the Fermi level, so once $C_{d}$ is known, $N_{t}$ can be calculated using equation (6). The energy $E_{t}$ of the deep level can be determined from the temperature dependence of the peak frequency $\omega_{p}$ by taking the slope of the Arrhenius plot. Once the energy is known, it can be plugged into equation (11) along with $\omega_{p}$ to determine the majority carrier capture cross section.

Good agreement is observed when the space charge depth determined from equation (9) is compared to the collected charge of a corresponding detector, indicating that both sensors are depleting from the front side. If any of these have gone through type inversion, it could imply presence of a double junction $[2,3]$.

\section{REFERENCES}

[1] M.K. Petterson, et al., RRESMDD06, Nucl. Inst. Meth. A (2007), doi:10.1016/j.nima.2007.08.222

[2] V. Chiochia, M. Swartz, "Simulation of Heavily Irradiated Pixel Sensors and Comparison with Test Beam Measurements." IEEE Nucl. Sci. Symposium, Oct. 20

[3] E. Verbitskaya et al., RESMDD07, Nucl. Inst. Meth. A (2007), in print.

[4] Yao WM et al., Review of Particle Physics, Chapter 27, J. Phys. G: Nucl. Part. Phys., 33 (2006) 265-267.

[5] G. Casse et al., Nucl. Inst. Meth. A 518 (2004) 340-342.

[6] M. Bruzzi et al., Nucl. Inst. Meth. A 552 (2005) 20-26.

[7] E. H. Nicollian and J.R. Brews, "MOS (Metal Oxide Semiconductor) Physics and Technology", 2003 
[8] M. Beguwala and C. R. Crowell, "Characterization of Multiple Deep Level Systems in Semiconductor Junctions by Admittance Measurements", Solid-State Electronics, (1974), Vol.1 7, pp. 203-214

[9] M. P. Verkhovodov, H. P. Peka, D. A. Pulemyotov, "Capacitance Behavior of Junctions with Frozen Dopant Levels", Semicond. Sci. Technol. 8 (1993) $1842-1847$.

[10] M.K. Petterson et al., "Determination of the Charge Collection Efficiency in Neutron Irradiated Silicon Detectors." SCIPP Preprints SCIPP 08/09 